\title{
Consumo de alimentos y asma en niños escolares de Cuernavaca
}

\author{
Rosa Inés Gutiérrez-Delgado, $M$ en $C$,(I) Albino Barraza-Villarreal, $M$ en $C$, Dr en $C$,(I) \\ María Consuelo Escamilla-Núñez, M en C, (I) Maritsa Solano-González, Lic en Inf, (I) Hortensia Moreno-Macías, M en C, (I) \\ Isabelle Romieu, MD, MSP, ScD.(I)
}

\section{Gutiérrez-Delgado RI, Barraza-Villarreal A, Escamilla-Núñez MC, Solano-González M, Moreno-Macías H, Romieu I. \\ Consumo de alimentos y asma en niños escolares de Cuernavaca. Salud Publica Mex 2009;5 I:202-2II.}

\section{Resumen}

Objetivo. Evaluar la relación entre la dieta y el desarrollo de asma y rinitis alérgica en escolares de Cuernavaca, Morelos, México. Material y métodos. Se aplicó el cuestionario ISAAC (International Study of Asthma and Allergies in Childhood) a 5460 niños de entre 6 a 8 y II a I 4 años de edad, de nivel primaria, seleccionados de manera aleatoria. Se formaron seis grupos de alimentos a partir de un análisis de clusters jerárquico y se evaluó la relación con los padecimientos a través de modelos de regresión logística. Resultados. El consumo de comida rápida tuvo un efecto adverso sobre la sibilancia actual $(R M=I .82$; IC95\%=I. I6-2.87) y el consumo de golosinas sobre más de tres episodios de sibilancia $(R M=2.26 ;$ IC95\%=I.04-4.95) y síntomas nasales sin gripa o catarro $(R M=I .35$; IC95\%=I.06-I.7I). Conclusiones. Este estudio provee evidencia de que la dieta juega un papel muy importante, ya que el consumo abundante de comida rápida y golosinas incrementa el riesgo de asma y rinitis.

Palabras clave: asma; rinitis; ISAAC; dieta; escolares; México
Gutiérrez-Delgado RI, Barraza-Villarreal A, Escamilla-Núñez MC, Solano-González M, Moreno-Macías H, Romieu I.

Food consumption and asthma in school children

in Cuernavaca, Morelos, Mexico.

Salud Publica Mex 2009;5 I:202-2I I.

\section{Abstract}

Objective. To evaluate the relation between diet and the development of asthma and allergic rhinitis in schoolchildren from Cuernavaca, Morelos, Mexico. Material and Methods. We apply the ISAAC's questionnaire in 5460 schoolchildren from 6 to 8 and II-I4-year-old of elementary level selected in random form. Six groups of food were formed using a hierarchic clusters analysis and the association was evaluated using logistic regression models. Results. The consumption of fast food had an adverse effect for current wheezing $(O R=1.82$; $C 195 \%=I .16-2.87)$ and the consumption of tidbits for more than three episodes of wheezing $(O R=2.26 ; C 195 \%=1.04-4.95)$ and nasal symptoms without cold, OR=I.35 (IC95\%; I.06-I.7I). Conclusions. This study provides evidence that the diet plays a very important role since the high consumption of fast food and tidbits increased the risk of asthma and rhinitis symptoms.

Key words: asthma; rhinitis; ISAAC; diet; schoolchildren; Mexico

(I) Instituto Nacional de Salud Pública. Cuernavaca, Morelos, México.

Fecha de recibido: 24 de marzo de 2008 - Fecha de aceptado: 4 de marzo de 2009 Solicitud de sobretiros: Dra. Isabelle Romieu. Instituto Nacional de Salud Pública. Av. Universidad 655, col. Santa María Ahuacatitlán. 62508 Cuernavaca, Morelos, México.

Correo electrónico: iromieu@correo.insp.mx 
$E^{1}$ asma, la rinitis y el eccema, llamados con frecuencia enfermedades atópicas, constituyen un importante problema de salud pública. En los últimos 20 años, las prevalencias de dichas anomalías se han incrementado en grado notable. ${ }^{1}$ Se calcula que en el mundo hay alrededor de 300 millones de personas con asma y se atribuyen a esta afección 180000 muertes cada año. El costo por tratamiento de este padecimiento varía en los diferentes países entre 300 y 1300 dólares por año. En Estados Unidos de América (EUA) el asma se presenta en casi $10 \%$ de la población. ${ }^{2}$

En México, en 2004 el asma se ubicó dentro de las primeras 20 causas de enfermedad. Del total de casos registrados, $28.5 \%$ se presentó en el grupo de 5 a 14 años de edad (población escolar). En el estado de Morelos el asma se encuentra entre las primeras causas de morbilidad, con una prevalencia de $35.3 \%$ de casos para el mismo grupo de edad. ${ }^{3}$

Este tipo de enfermedades tiene, además, un amplio impacto sobre los niveles de actividad de niños y adolescentes y altera de manera notoria su calidad de vida. ${ }^{4}$ Las causas de dicho trastorno no son claras. Entre los factores investigados al respecto figuran los relacionados con las condiciones de salud, vivienda, estilos de vida y alimentación, además de los vinculados con factores ambientales (humedad, convivencia con animales, contaminación). ${ }^{5-13}$

Los resultados de estudios epidemiológicos que evalúan la relación entre asma y dieta no han sido concluyentes y se han reportado hallazgos controversiales. Por un lado se ha encontrado que ciertos componentes de la dieta (carnes rojas y mantequilla) se relacionan con el incremento de la prevalencia de asma y rinitis alérgica; ${ }^{14-16}$ otros estudios han mencionado que personas con alto consumo de fruta fresca, ${ }^{17-20}$ vegetales, ${ }^{17,18,21,22}$ productos elaborados con granos enteros, ${ }^{23}$ pescado ${ }^{15,24,25}$ y leche entera o de granja ${ }^{22,23,26}$ tienen baja prevalencia; asimismo, se ha señalado que un mayor consumo de ácidos grasos poliinsaturados omega 6 favorece un incremento del riesgo. Por otro lado, un elevado consumo de ácidos grasos omega 3 se vincula de forma inversa. ${ }^{27,28}$

Debido a lo anterior, se realizó un análisis secundario del estudio general sobre asma y enfermedades alérgicas en la ciudad de Cuernavaca, Morelos, México, bajo la metodología de la tercera fase del International Study of Asthma and Allergies in Childhood (ISAAC, por sus siglas en inglés $)^{29}$ con el propósito de determinar el patrón de dieta y la influencia de éste sobre la prevalencia de tales enfermedades en niños escolares participantes en el estudio.

\section{Material y métodos}

El estudio se llevó a cabo en Cuernavaca, Morelos, México, en 2002, con base en la tercera fase del protocolo ISAAC. ${ }^{30}$ Recibió aprobación de las diferentes comisiones del Instituto Nacional de Salud Pública (Ética, Bioseguridad e Investigación). El protocolo ISAAC se estableció para maximizar el valor de la investigación epidemiológica de estas anomalías alérgicas.

El marco muestral estuvo conformado por 137 escuelas primarias, tanto públicas como privadas, registradas en la Secretaría de Educación Pública del estado de Morelos. El universo de estudio se integró con el total de niños inscritos en dichas escuelas. En el cálculo del tamaño de la muestra se siguieron los lineamientos del ISAAC, que recomiendan un tamaño de muestra de 7200 niños en total, 3600 por cada grupo de edad ( 6 a 8 y 11 a 14 años), más 20\% de posibles pérdidas. Para el caso del presente estudio, el tamaño de la muestra final se conformó con 5460 niños, que asistían a las 72 escuelas primarias, seleccionadas de manera aleatoria desde la primera fase del proyecto. Se incluyó en el estudio a todos los niños que asistieron a los grupos de primero y sexto grados, que estuvieran dentro de los grupos de edad mencionados y que contaran con el consentimiento por escrito de sus padres. Al final, 4970 (91\%) participantes se incluyeron en el análisis. Tatto y colaboradores publicaron los detalles de la metodología. ${ }^{31}$

En cuanto a la recolección de la información, se utilizó el cuestionario estandarizado y validado por ISAAC, ${ }^{32}$ adaptado para su uso en México, el cual ya se empleó con anterioridad en otras investigaciones. ${ }^{13,31,33}$ El llenado del cuestionario lo llevaron a cabo los padres de los participantes para ambos grupos de edad, por un mecanismo de autoadministración. El cuestionario incluyó preguntas sobre sibilancias en los últimos 12 meses, sibilancias sin gripa o catarro y más de tres episodios de sibilancias en los últimos 12 meses, así como sobre la ocurrencia de síntomas relacionados con rinitis alérgica, síntomas nasales sin gripa o catarro y síntomas nasales más síntomas oculares. Asimismo, se interrogó sobre otros posibles factores de riesgo, aspectos relacionados con el diagnóstico y tratamiento médico de estas afecciones, historial médico del niño y antecedentes de problemas de tipo respiratorio. De forma adicional se incorporaron preguntas relacionadas con la frecuencia de consumo de alimentos en los últimos 12 meses.

En este sentido, respecto al interrogatorio sobre el consumo de alimentos, se incluyeron 32 preguntas para evaluar el consumo de frutas y verduras, grasas de origen animal y vegetal, lácteos, cereales, pastas, 
pescados y mariscos y suplementos como calcio, hierro y vitaminas. En cuanto a la frecuencia, se evaluó el consumo semanal mediante una escala de tres niveles: nunca/ocasionalmente, 1 a 2 veces por semana y casi todos los días.

\section{Análisis estadístico}

Se exploró la matriz de correlación de las variables relacionadas con la dieta, para evaluar en qué proporción estaban intercorrelacionadas. Se observaron varios grupos de alimentos claramente definidos, como mariscos y pescados, frutas y verduras, pastas y arroz, oleaginosas y comida chatarra. Después se efectuó una exploración más minuciosa a partir del análisis de clusters jerárquico con el objetivo de reconocer agrupaciones más definidas de alimentos.

Se identificaron seis agrupaciones de alimentos: "frutas y verduras" (uvas, durazno, brócoli y zanahoria), "oleaginosas" (nuez, avellana, cacahuate y almendra), "pescados y mariscos" (mariscos, pescados y pescado en lata), "golosinas" (papas fritas y chocolates), "queso y crema" y "pasta y arroz". Se incluyó también la comida rápida como alimento independiente, ya que no se clasificó dentro de ningún grupo. Se generó una variable por grupo de alimento a partir de las combinaciones de los alimentos incluidos en cada grupo. Se recategorizó cada variable generada de acuerdo con su distribución y se utilizaron dos o tres categorías, de acuerdo con la frecuencia de consumo: bajo, moderado y frecuente.

Se corrieron modelos logit para evaluar la relación entre cada una de las agrupaciones de alimentos (en sus diferentes categorías y mediante el consumo bajo como categoría de referencia) y la presencia de síntomas respiratorios (sibilancias, sibilancias sin gripa o catarro, más de tres episodios de sibilancias, síntomas nasales sin gripa o catarro, síntomas nasales más síntomas oculares), con ajuste por variables de confusión, como edad, sexo, tabaquismo pasivo durante el primer año de vida, infecciones respiratorias durante el primer año de vida y antecedentes familiares de atopia. También se corrieron modelos tras considerar los diferentes grupos de alimentos como variables independientes y se ajustó por variables de confusión.

\section{Resultados}

De los 4970 niños incluidos en el análisis, 49.5\% tenía entre 6 y 8 años de edad y 50.5\% entre 11 y 14 años. Respecto a la escolaridad de la madre, $3.98 \%$ no contaba con algún nivel educativo, 25.22\% tenía educación primaria, $34.55 \%$ educación secundaria, $19.54 \%$ educación media superior (preparatoria) y $16.71 \%$ educación superior.

La prevalencia de asma por diagnóstico médico fue de 9.19\% (IC95\%:8.38-10.01). La presencia de sibilancias, sibilancias sin gripa o catarro y más de tres episodios de sibilancia en los últimos 12 meses fue de 8.09\% (IC95\%:7.32-8.86), 2.64\% (IC95\%:2.18-3.10) y $1.8 \%$ (IC95\%:1.42-2.18), respectivamente. Las prevalencias encontradas, relacionadas con síntomas de rinitis alérgica, se pueden observar en el cuadro I.

El cuadro II presenta la frecuencia de consumo de alimentos en esta población. Destaca el bajo consumo de pescados y mariscos, oleaginosas, suplementos, mantequilla y comida rápida; y, por otro lado, el elevado consumo de fruta, pasta, arroz, pan y cereal.

Cuadro I

Prevalencia de ASma y RiNitis Por diagnóstico médico y Síntomas en Niños escolares de Cuernavaca, Morelos, México, 2002

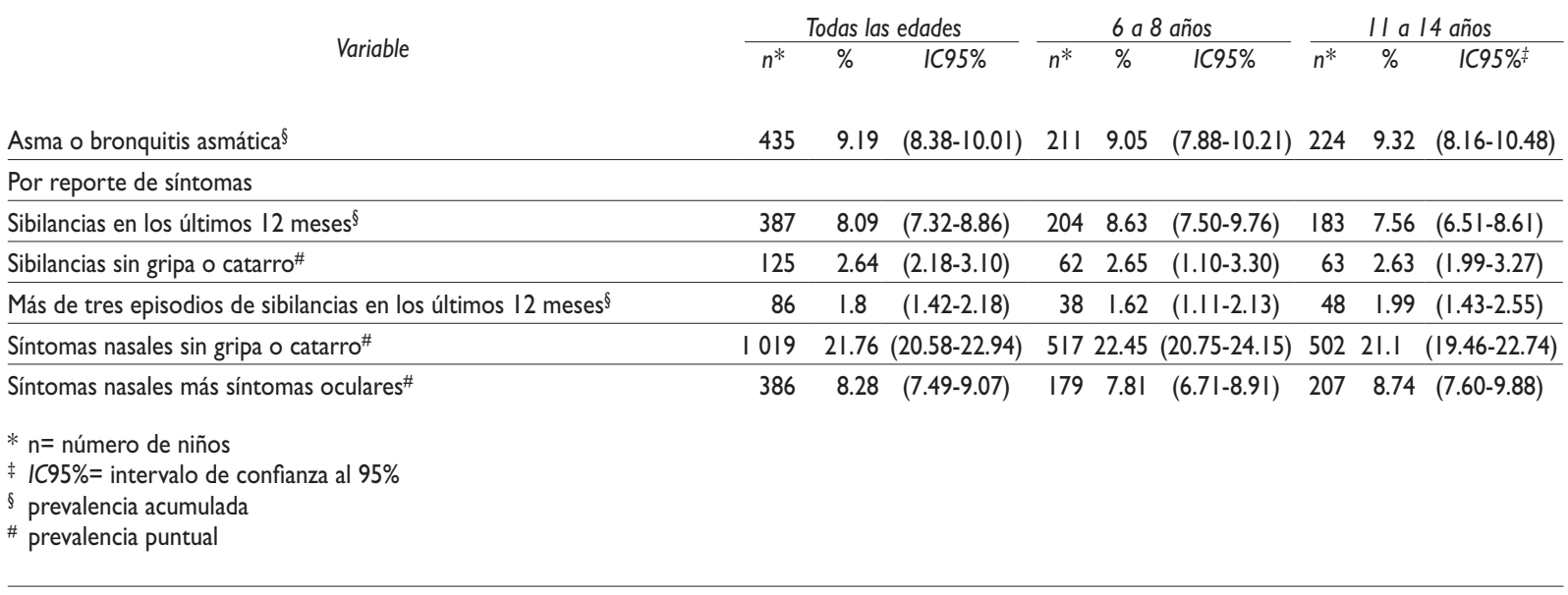


Cuadro II

Frecuencia de consumo de alimentos en niños escolares de Cuernavaca, Morelos, México, 2002

\begin{tabular}{|c|c|c|c|c|}
\hline \multirow{2}{*}{ Alimentos } & \multicolumn{3}{|c|}{ Consumo por semana (\%) } & \multirow{2}{*}{ Total de observaciones } \\
\hline & Nuncalocasionalmente & I o 2 veces por semana & Casi todos los días & \\
\hline Fruta* & 8.41 & 32 & 59.59 & 4937 \\
\hline Verdura $^{\ddagger}$ & 17.93 & 42.52 & 39.54 & 4868 \\
\hline Pescado y mariscos ${ }^{\S}$ & 72.57 & 25.79 & 1.63 & 4711 \\
\hline Huevo & 24.48 & 60.53 & 14.98 & 4865 \\
\hline Lácteos\# & 20.98 & 44.10 & 34.92 & 4891 \\
\hline Crema, margarina & 30.76 & 42.15 & 27.09 & 4747 \\
\hline Mantequilla & 61.95 & 26.47 & 11.59 & 4436 \\
\hline Cereal & 20.61 & 32.93 & 46.46 & 4746 \\
\hline Pasta, arroz, pan & 7.57 & 30.32 & 62.11 & 4864 \\
\hline Oleaginosas $^{\&}$ & 75.97 & 18.24 & 5.79 & 4561 \\
\hline Comida chatarra $^{*}$ & 38.04 & 35.67 & 26.29 & 4645 \\
\hline Comida rápida & 77.47 & 15.95 & 6.58 & 4269 \\
\hline Suplementos & 64.49 & 14.89 & 20.62 & 4365 \\
\hline \multicolumn{5}{|c|}{ * Naranja, uvas, plátano y durazno o chabacano } \\
\hline \multicolumn{5}{|c|}{ ‡ Brócoli, coliflor, jitomate y zanahoria } \\
\hline \multicolumn{5}{|c|}{$\S$ Pescados, mariscos y lata de pescado } \\
\hline \multicolumn{5}{|c|}{ \# Leche y queso } \\
\hline \multicolumn{5}{|c|}{ \& Almendra, nuez, avellana y cacahuate } \\
\hline${ }^{*}$ Dulces, chocolates y pa & & & & \\
\hline
\end{tabular}

En cuanto al análisis bivariado, algunos de los principales factores que se vincularon con el riesgo de sibilancias y rinitis fueron: antecedentes familiares de asma ( $R M=2.64$; IC95\%:2.03-3.40) e infecciones respiratorias durante el primer año de vida $(R M=3.13$; IC95\%:2.48-3.95); y, en relación con la escolaridad materna, la probabilidad de tener asma y síntomas de asma fue menor conforme se incrementaba el nivel educativo, situación no observada para la rinitis y los síntomas nasales; empero, estas vinculaciones no fueron significativas (cuadro III).

Al relacionar las variables de respuesta con los alimentos estudiados, ajustados por potenciales de confusión, se observó que para las sibilancias en los últimos 12 meses hubo un efecto protector con el consumo frecuente de frutas y verduras $(R M=0.71 ; I C 95 \%: 0.52-0.97)$, el consumo moderado de queso y crema $(R M=0.73$; IC95\%:0.55-0.98) y el consumo frecuente de oleaginosas ( $R M=0.70 ;$ IC95\%:0.51-0.96) (cuadro IV).

En relación con la presencia de más de tres episodios de sibilancias en los últimos 12 meses, se reconoció un efecto protector del consumo moderado de frutas y verduras ( $R M=0.40$; IC95\%:0.20-0.80), el consumo frecuente de pescados y mariscos $(R M=0.36$; IC95\%:0.16-0.80) y el consumo moderado de queso y crema ( $R M=0.54$; IC95\%;0.29-0.99).

En cuanto a los síntomas nasales sin gripa o catarro, el consumo frecuente de frutas y verduras y el consumo moderado de oleaginosas tuvieron un efecto protector ( $R M=0.73$; IC95\%:0.59-0.90; y $R M=0.74 ;$ IC95\%;0.61-0.90, respectivamente).

Para los síntomas nasales más síntomas oculares, el consumo frecuente de frutas y verduras redujo la probabilidad de presentar síntomas ( $R M=0.57$; IC95\%: 0.42-0.79).

En cuanto al análisis multialimento, después de ajustar por potenciales confusores, se observó que el consumo moderado y frecuente de frutas y verduras se relacionó con un efecto protector para sibilancias en los últimos 12 meses ( $R M=0.74$; IC95\%:0.56-0.99; $R M=0.68$; IC95\%;0.49-0.93, respectivamente), y, por el contrario, el consumo frecuente de comida rápida tuvo un efecto adverso ( $R M=1.82$; IC95\%:1.16-2.87). Asimismo, se advirtió que el consumo frecuente de golosinas elevó la probabilidad de presentar más de tres episodios de sibilancias (RM=2.26; IC95\%:1.04-4.95) (cuadro V).

Para la rinitis alérgica, el consumo frecuente de frutas y verduras se relacionó con un efecto protector 
Cuadro III

Consumo de alimentos, asma y rinitis en niños escolares de Cuernavaca, Morelos México, 2002. ANÁLISIS BIVARIADO

\begin{tabular}{|c|c|c|c|c|c|c|c|c|c|c|c|}
\hline \multirow[t]{2}{*}{ Grupo de alimentos } & \multirow[t]{2}{*}{$n$} & \multicolumn{2}{|c|}{$\begin{array}{c}\text { Sibilancias en los últimos } \\
12 \text { meses }\end{array}$} & \multicolumn{2}{|c|}{$\begin{array}{l}\text { Sibilancias sin } \\
\text { gripa o catarro }\end{array}$} & \multicolumn{2}{|c|}{$\begin{array}{c}\text { Más de tres episodios de } \\
\text { sibilancias en los últimos } \\
12 \text { meses }\end{array}$} & \multicolumn{2}{|c|}{$\begin{array}{l}\text { Sintomas nasales sin } \\
\text { gripa o catarro }\end{array}$} & \multicolumn{2}{|c|}{$\begin{array}{c}\text { Sintomas nasales más } \\
\text { síntomas } \\
\text { oculares }\end{array}$} \\
\hline & & $R M^{*}$ & IC95\% & $R M^{*}$ & IC95\% & $R M^{*}$ & IC95\% $\%^{\ddagger}$ & $R M^{*}$ & IC95\% $\%^{\ddagger}$ & $R M^{*}$ & IC95\% $\%^{\ddagger}$ \\
\hline \multicolumn{12}{|l|}{ Fruta y verduras ${ }^{\S}$} \\
\hline Bajo\# & 1.675 & 1.00 & & 1.00 & & 1.00 & & 1.00 & & 1.00 & \\
\hline Moderado & 1.353 & 0.78 & $0.60-1.01$ & 0.80 & $0.51-1.25$ & 0.57 & $0.32-1.02$ & 0.99 & $0.83-1.75$ & 0.85 & $0.66-1.10$ \\
\hline Frecuente & 1.139 & 0.69 & $0.52-0.93$ & 0.75 & $0.46-I .2 I$ & 0.63 & $0.35-1.15$ & 0.70 & $0.58-0.85$ & 0.60 & $0.45-0.81$ \\
\hline \multicolumn{12}{|l|}{ Pescados y mariscos ${ }^{\&}$} \\
\hline Bajo & 2.789 & 1.00 & & 1.00 & & 1.00 & & 1.00 & & 1.00 & \\
\hline Frecuente & 1.097 & 0.84 & $0.64-1.01$ & 1.01 & $0.66-0.56$ & 0.51 & $0.26-.97$ & 0.95 & $0.80-1.13$ & 0.90 & $0.69-1.16$ \\
\hline \multicolumn{12}{|l|}{ Queso y crema } \\
\hline Bajo\# & 1.404 & 1.00 & & 1.00 & & 1.00 & & 1.00 & & 1.00 & \\
\hline Moderado & 1.592 & 0.81 & $0.62-1.05$ & 0.79 & $0.51-1.23$ & 0.56 & $.032-0.98$ & 1.01 & $0.84-1.20$ & 1.02 & $.78-1.33$ \\
\hline Frecuente & 1.563 & 0.87 & $0.67-I .12$ & 0.74 & $0.47-I .16$ & 0.77 & $0.46-1.30$ & 0.99 & $0.83-1.18$ & 1.00 & $.77-1.31$ \\
\hline \multicolumn{12}{|l|}{ Pasta y arroz ${ }^{\&}$} \\
\hline Bajo\# & 1.173 & 1.00 & & 1.00 & & 1.00 & & 1.00 & & 1.00 & \\
\hline Moderado & 1.900 & 0.93 & $0.70-1.22$ & 0.85 & $0.53-1.37$ & 0.81 & $0.45-1.44$ & 1.00 & $0.8-1.20$ & 1.03 & $0.79-1.33$ \\
\hline Frecuente & 1.526 & 1.10 & $0.83-1.45$ & 1.19 & $0.75-1.90$ & 1.01 & $0.57-1.79$ & 0.91 & $0.75-1.10$ & 0.84 & $0.63-1.11$ \\
\hline \multicolumn{12}{|l|}{ Oleaginosas ${ }^{\ell, \neq}$} \\
\hline Bajo & 2.973 & 1.00 & & 1.00 & & 1.00 & & 1.00 & & 1.00 & \\
\hline Frecuente & 1.003 & 0.74 & $0.59-0.99$ & 0.55 & $0.32-0.92$ & 1.01 & $0.58-1.76$ & 0.77 & $0.64-0.92$ & 0.82 & $0.63-1.08$ \\
\hline \multicolumn{12}{|l|}{ Comida rápida } \\
\hline Bajo\# & 3.307 & 1.00 & & 1.00 & & 1.00 & & 1.00 & & 1.00 & \\
\hline Moderado & 681 & 1.10 & $0.82-1.48$ & 0.66 & $0.36-1.21$ & 0.47 & $0.20-.09$ & 1.00 & $0.82-1.23$ & 0.99 & $0.73-1.34$ \\
\hline Frecuente & 281 & 1.36 & $0.9-2.05$ & 1.50 & $0.79-2.85$ & 1.82 & $0.8-3.70$ & 1.09 & $0.82-1.47$ & I.II & $0.72-1.72$ \\
\hline \multicolumn{12}{|l|}{ Golosinas } \\
\hline Bajo\# & 1.537 & 1.00 & & 1.00 & & 1.00 & & 1.00 & & 1.00 & \\
\hline Moderado & 1.992 & 1.07 & $0.83-1.37$ & 0.91 & $0.59-1.39$ & 1.40 & $0.81-2.43$ & 1.16 & $0.98-1.37$ & 1.05 & $0.8-1.35$ \\
\hline Frecuente & 821 & 1.18 & $0.87-I .6 I$ & 1.18 & $0.71-1.96$ & 1.81 & $0.9-3.42$ & 1.22 & $0.99-1.49$ & $\mathrm{I} .4 \mathrm{I}$ & $1.05-1.90$ \\
\hline $\begin{array}{l}\text { * RM= razón de momios } \\
\text { ‡ IC95\%= intervalo de co } \\
\text { \& uva, durazno, brócoli y } \\
\text { \# categoría de referencia } \\
\text { \& variable dicotómica } \\
\text { * almendra, avellana, nuez }\end{array}$ & $\begin{array}{l}\text { anza al } 95 \% \\
\text { ahoria } \\
\text { cacahuate }\end{array}$ & & & & & & & & & & \\
\hline
\end{tabular}

para los síntomas nasales sin gripa o catarro $(R M=0.79$; IC95\%:0.63-0.99), de igual modo para los niños que refirieron mayor consumo de oleaginosas $(R M=0.74$; IC95\%:0.60-0.92). Los niños que informaron un mayor consumo de golosinas (moderado y frecuente) tuvieron mayor probabilidad de presentar síntomas nasales sin gripa o catarro en comparación con los niños que tenían bajo consumo ( $R M=1.23$; IC95\%:1.02-1.49; y $R M=1.35$; IC95\%;1.06-1.71, respectivamente).

Por ultimo, en relación con los síntomas nasales más síntomas oculares, el consumo frecuente de frutas y verduras tuvo un efecto protector $(R M=0.59$; IC $95 \%$ :0.42- 


\section{Cuadro IV}

Consumo de alimentos, y asma y rinitis en niños escolares de Cuernavaca, Morelos, México, 2002.

ANÁLISIS MULTIVARIAdO

\begin{tabular}{|c|c|c|c|c|c|c|}
\hline \multirow[t]{2}{*}{ Grupo } & \multirow[t]{2}{*}{$n$} & $\begin{array}{c}\text { Sibilancias en los últimos } \\
\qquad 12 \text { meses }\end{array}$ & $\begin{array}{l}\text { Sibilancias sin } \\
\text { gripa o catarro }\end{array}$ & $\begin{array}{c}\text { Más de tres episodios de } \\
\text { sibilancias en los últimos } \\
12 \text { meses }\end{array}$ & $\begin{array}{l}\text { Sintomas nasales sin } \\
\text { gripa o catarro }\end{array}$ & $\begin{array}{c}\text { Sintomas nasales más } \\
\text { sintomas } \\
\text { oculares }\end{array}$ \\
\hline & & IC95\%§ & IC95\%ई & IC95\%§ & IC95\%§ & IC95\%§ \\
\hline
\end{tabular}

Fruta y verduras ${ }^{\#}$

\begin{tabular}{llllllllllll} 
Bajo $^{\&}$ & 1.675 & $1.00^{*}$ & & 1.00 & & 1.00 & & $1.00^{*}$ & & $1.00^{*}$ \\
\hline Moderado & 1.353 & 0.76 & $0.58-1.01$ & 0.84 & $0.52-1.35$ & 0.40 & $0.20-0.80$ & 0.99 & $0.82-1.19$ & 0.78 & $0.60-1.03$ \\
\hline Frecuente & 1.139 & 0.71 & $0.52-0.97$ & 0.71 & $0.41-1.24$ & 0.64 & $0.33-1.23$ & 0.73 & $0.59-0.90$ & 0.57 & $0.42-0.79$
\end{tabular}

Pescados y mariscos ${ }^{\infty}$

\begin{tabular}{|c|c|c|c|c|c|c|c|c|c|c|c|}
\hline Bajo\& & 2.789 & 1.00 & & 1.00 & & 1.00 & & 1.00 & & 1.00 & \\
\hline Frecuente & 1.097 & 0.79 & $0.60-1.06$ & 1.02 & $0.64-1.62$ & 0.36 & $0.16-0.80$ & 0.92 & $0.76-1.10$ & 0.88 & $0.67-1.16$ \\
\hline
\end{tabular}

Queso y crema

\begin{tabular}{|c|c|c|c|c|c|c|c|c|c|c|c|}
\hline Bajo\& & 1.404 & 1.00 & & 1.00 & & 1.00 & & 1.00 & & 1.00 & \\
\hline Moderado & 1.592 & 0.73 & $0.55-0.98$ & 0.79 & $0.48-1.29$ & 0.54 & $0.29-0.99$ & 0.98 & $0.82-1.19$ & 0.97 & $0.73-1.29$ \\
\hline Frecuente & 1.563 & 0.80 & $0.60-1.07$ & 0.77 & $0.47-1.27$ & 0.62 & $0.35-1.12$ & 1.00 & $0.83-1.21$ & 0.98 & $0.73-1.30$ \\
\hline
\end{tabular}

Pasta $y$ arroz $z^{\infty}$

\begin{tabular}{lrrrrrrrrrrr} 
Bajo\& & 1.173 & 1.00 & & 1.00 & & 1.00 & & 1.00 & & 1.00 & \\
\hline Moderado & 1.900 & 0.92 & $0.68-1.23$ & .81 & $0.48-1.38$ & 0.81 & $0.44-1.50$ & 0.98 & $0.81-1.19$ & 0.98 & $0.74-1.30$ \\
\hline Frecuente & 1.526 & 1.04 & $0.7-1.41$ & 1.25 & $0.75-2.11$ & 0.90 & $0.48-1.70$ & 0.87 & $0.71-1.07$ & 0.80 & $0.59-1.09$
\end{tabular}

Oleaginosas $^{\infty, \varnothing}$

\begin{tabular}{|c|c|c|c|c|c|c|c|c|c|c|c|}
\hline Bajo $^{\&}$ & 2.973 & 1.00 & & 1.00 & & 1.00 & & 1.00 & & 1.00 & \\
\hline Frecuente & 1.003 & 0.70 & $0.5 I-0.96$ & 0.57 & $0.32-1.00$ & 0.86 & $0.46-1.6 I$ & 0.74 & $0.6 \mid-0.90$ & 0.75 & $0.55-1.01$ \\
\hline
\end{tabular}

Comida rápida

\begin{tabular}{lccccccccccc} 
Bajo\& & 3.307 & 1.00 & & 1.00 & & 1.00 & & 1.00 & & 1.00 \\
\hline Moderado & 681 & 1.12 & $0.81-1.54$ & 0.74 & $0.40-1.39$ & 0.55 & $0.23-1.30$ & 1.01 & $0.81-1.26$ & 0.94 & $0.68-1.31$ \\
\hline $\begin{array}{l}\text { Frecuente } \\
\text { Golosinas }\end{array}$ & 281 & 1.43 & $0.92-2.24$ & 1.66 & $0.83-3.32$ & 1.98 & $0.92-4.27$ & 1.08 & $0.78-1.50$ & 1.18 & $0.74-1.87$ \\
Bajo\& & & & & & & & & & & & \\
\hline Moderado & 1.537 & 1.00 & & 1.00 & & 1.00 & & 1.00 & & 1.00 & \\
\hline Frecuente & 1.992 & 1.00 & $0.77-1.31$ & 0.88 & $0.55-1.41$ & 1.44 & $0.79-2.64$ & 1.12 & $0.93-1.33$ & 0.94 & $0.72-1.23$ \\
\hline & 821 & 1.046 & $0.75-1.47$ & 1.13 & $0.65-1.98$ & 1.64 & $0.81-3.34$ & 1.17 & $0.94-1.47$ & 1.32 & $0.96-1.82$
\end{tabular}

* $R M=$ razón de momios

‡ ajustado por: sexo, tabaquismo pasivo durante el primer año de vida, antecedente familiar de atopia, infecciones respiratorias durante el primer año de vida y edad

§ IC95\%= intervalo de confianza al $95 \%$

\# uvas, durazno, brócoli y zanahoria

\& categoría de referencia

* est de tendencia $p<0.05$

${ }^{\infty}$ variable dicotómica

- almendras, avellanas, nuez y cacahuate 


\title{
CuadroV
}

Consumo de alimentos, y asma y rinitis en niños escolares de Cuernavaca, Morelos, México, 2002. ANÁLISIS MULTIALIMENTO

Variable

\author{
$n$
}

$R M^{*}$

$1 C 95 \%$

Sibilancia en los últimos 12 meses*

Fruta y verduras

\begin{tabular}{llll} 
Bajo & 1.675 & 1.00 & \\
\hline Moderado & 1.353 & 0.74 & $0.56-0.99$ \\
\hline $\begin{array}{l}\text { Frecuente } \\
\text { Comida rápida } \\
\text { Bajo }\end{array}$ & 1.139 & 0.68 & $0.49-0.93$ \\
\hline Moderado & 3.307 & & \\
\hline Frecuente & 681 & 1.00 & $0.75-1.49$ \\
\hline
\end{tabular}

Sibilancias sin gripa o catarro

\section{Oleaginosas}

\begin{tabular}{lccc} 
Bajo & 2.973 & 1.00 & \\
\hline $\begin{array}{l}\text { Frecuente } \\
\text { Comida rápida } \\
\text { Bajo }\end{array}$ & 1.003 & 0.56 & $0.32-0.99$ \\
\hline Moderado & 3.307 & & 1.00 \\
\hline Frecuente & 681 & 0.87 & $0.46-1.64$ \\
\hline
\end{tabular}

Más de tres episodios de sibilancias en los últimos 12 meses $\$$

Pescados y mariscos

\begin{tabular}{|c|c|c|c|}
\hline Bajo & 2.789 & 1.00 & \\
\hline Frecuente & 1.097 & 0.34 & $0.14-0.82$ \\
\hline \multicolumn{4}{|l|}{ Golosinas } \\
\hline Bajo & 1.537 & 1.00 & \\
\hline Moderado & 1.992 & 1.52 & $0.76-3.07$ \\
\hline Frecuente & 821 & 2.26 & I.04-4.95 \\
\hline
\end{tabular}

Síntomas nasales sin gripa o catarro*

Fruta y verduras

\begin{tabular}{|c|c|c|c|}
\hline Bajo & 1.675 & 1.00 & \\
\hline Moderado & 1.353 & 0.99 & $0.82-1.21$ \\
\hline Frecuente $^{\ddagger}$ & 1.139 & 0.79 & $0.63-0.99$ \\
\hline \multicolumn{4}{|l|}{ leaginosas } \\
\hline Bajo & 2.973 & 1.00 & \\
\hline Frecuente & 1.003 & 0.74 & $0.60-0.92$ \\
\hline \multicolumn{4}{|l|}{ olosinas } \\
\hline Bajo & 1.537 & 1.00 & \\
\hline Moderado & 1.992 & 1.23 & $1.02-1.49$ \\
\hline Frecuente ${ }^{\ddagger}$ & 821 & 1.35 & $|.06-1.7|$ \\
\hline
\end{tabular}

Síntomas nasales más síntomas oculares\#

Fruta y verduras

\begin{tabular}{lccc} 
Bajo & 1.675 & 1.00 & \\
\hline Moderado & 1.353 & 0.79 & $0.60-1.04$ \\
\hline $\begin{array}{l}\text { Frecuente } \\
\text { Golosinas }\end{array}$ & 1.139 & 0.59 & $0.42-0.82$ \\
Bajo & & & \\
Moderado & 1.537 & 1.00 & $0.80-1.40$ \\
\hline Frecuente & 1.992 & 1.06 & $1.12-2.14$
\end{tabular}

$R M=$ razón de momios

* modelo ajustado por: tabaquismo pasivo durante el primer año de vida, sexo, antecedente familiar de atopia, edad

¥ modelo ajustado por: sexo, tabaquismo pasivo durante el primer año de vida, antecedente familiar de atopia, consumo de pasta y arroz, edad

$\S$ modelo ajustado por: sexo, tabaquismo pasivo durante el primer año de vida, infección respiratoria durante el primer año de vida, consumo de queso y crema, edad

\# modelo ajustado por: sexo, tabaquismo pasivo durante el primer año de vida, edad

\& Prueba de tendencia: $p<0.05$ 
0.82) y el consumo frecuente de golosinas se vinculó con una mayor probabilidad de desarrollarlos $(R M=1.55$; IC95\%:1.12-2.14).

\section{Discusión}

Los resultados señalan que el consumo de comida rápida y golosinas incrementa la probabilidad de presentar sibilancias y síntomas nasales en los últimos 12 meses, y que el consumo de oleaginosas, frutas, verduras, pescados y mariscos favorece la disminución de la prevalencia de los padecimientos investigados, tal como se ha informado en otras investigaciones.

El efecto protector del consumo de frutas y vegetales se ha reportado en diferentes estudios. ${ }^{34,35}$ Los vegetales que poseen un alto contenido de vitaminas y minerales (magnesio, potasio, hierro y fibras) se han relacionado inversamente con la prevalencia de asma en adultos ${ }^{18} \mathrm{y}$ niños. ${ }^{22}$

El efecto protector que tienen frutas y verduras frente al asma y el desencadenamiento de síntomas respiratorios se debe a su alto contenido de antioxidantes, ya que son ricas en vitaminas $\mathrm{A}, \mathrm{E}$ y $\mathrm{C}$.

Se ha observado que los antioxidantes son la primera línea de defensa contra los radicales libres, los cuales son capaces de dañar los componentes celulares y contribuir a la inflamación. Asimismo, se ha señalado que los lignanos ${ }^{36} \mathrm{y}$ flavonoides tienen una gran capacidad para neutralizar los radicales libres y de estos últimos el resveratrol (presente en cacahuates, nueces y sobre todo en uvas) tiene propiedades antiinflamatorias debido a su efecto inhibidor sobre la ciclooxigenasa. ${ }^{37} \mathrm{La}$ vitamina E (que es liposoluble) y la vitamina C (hidrosoluble y muy abundante en el líquido extracelular pulmonar) representan una de las principales defensas del cuerpo contra los efectos de la oxidación, ya que contrarrestan dicho proceso a través de varios mecanismos. De igual modo, el caroteno $\beta$, precursor de la vitamina $\mathrm{A}$, acumulado en las membranas tisulares, genera aniones superóxidos, los cuales reaccionan de forma directa con la peroxidación de los radicales libres. ${ }^{38}$

En cuanto al efecto protector de las oleaginosas (nuez, almendra, cacahuate y avellana), se ha observado que aportan al organismo grasa de origen vegetal y son la principal fuente de vitamina E, además de que interactúan con radicales libres solubles en lípidos de la membrana celular. De esta forma mantienen la integridad de ésta y confieren protección a las células ante la presencia de compuestos tóxicos, metales pesados (plata, mercurio, plomo), drogas y radiaciones. De igual manera, inducen la proliferación de células de defensa y aumentan la respuesta celular ante algún daño o in- fección. Troisi y colaboradores encontraron que el alto consumo de vitamina E proveniente de la dieta tiene un efecto protector en la incidencia de asma en mujeres de mediana edad; sin embargo, este nexo podría estar relacionado con el hecho de que las personas alérgicas no comen este tipo de alimentos, como la nuez, dado que actúa como posible alergeno. ${ }^{39}$

También se ha reconocido que la infección y la inhalación de contaminantes ambientales activan los procesos oxidativos, por lo que el consumo de antioxidantes puede actuar fundamentalmente en el equilibrio de la respuesta al estrés oxidativo y la evolución del asma, al modular el impacto de los oxidantes en los pulmones y atenuar la inflamación de la vía respiratoria. ${ }^{38}$

Por otro lado, se ha comunicado que el magnesio que se encuentra en los vegetales de hojas verdes, nuez y granos enteros es esencial para el mantenimiento del potencial eléctrico a través de la membrana celular y por tanto modula el tono y el diámetro bronquiales. ${ }^{40}$

El efecto protector mostrado por el consumo de mariscos y pescado para la intensidad del asma (más de tres episodios de sibilancias en los últimos 12 meses) ya se ha reportado en otros estudios realizados en niños. ${ }^{24,25,41}$ Dicho efecto puede atribuirse al elevado contenido de proteínas, como lisina y triptófano; vitaminas, como A, D, E, complejo B, incluidas tiamina, riboflavina, niacina, vitamina B6 y B12, así como ácido pantoténico; minerales como calcio, fósforo, potasio, hierro, cobre, cobalto y otros; y ácidos grasos poliinsaturados omega 3 (n-3 PUFA). ${ }^{42}$ Los n-3 PUFA se almacenan en las membranas de las células y tienen una importante función en el control de la inflamación, pero pueden reemplazarse con facilidad por los ácidos grasos poliinsaturados omega 6 (n-6 PUFA). ${ }^{38}$ El consumo de n-6 PUFA es promotor de la formación de prostaglandina E2 (PGE2), conocida por acelerar la formación de IgE dentro de las células T. Los ácidos n-3 PUFA, ácido eicosapentanoico (EPA) y decosahexanoico (DHA), contenidos en el aceite de pescado, compiten e inhiben la formación de prostaglandinas y leucotrienos, derivados del ácido araquidónico, y suprimen el efecto sobre la función de los neutrófilos, de donde se desprende su potencial antiinflamatorio. ${ }^{15}$

La comida rápida o fast food, definida por el Departamento de Agricultura de EUA como "comida adquirida en autoservicios o kioscos al paso", y las papas fritas tienden a ser alimentos ricos en grasas, con alta densidad energética y pobre en micronutrientes y fibras. Los autores encontramos que el consumo de comida rápida aumenta la probabilidad de presentar sibilancias en los últimos12 meses. Estos resultados coinciden con los de Huang y colaboradores, en un trabajo desarrollado con adolescentes de Taiwán. ${ }^{14} \mathrm{El}$ chocolate tiene un 
alto contenido de antioxidantes y flavonoides, aunque también se ha reportado como alimento que provoca hipersensibilidad..$^{43}$

Es importante señalar que el jitomate y la naranja, ricos en vitaminas y antioxidantes, son alimentos que por sus características de accesibilidad son consumidos con mayor frecuencia por esta población, si bien en el análisis estadístico no se correlacionaron con las demás variables del grupo de frutas y verduras.

Hay que mencionar algunos aspectos metodológicos que tal vez influyeron en la interpretación de los hallazgos. En primer lugar, en este estudio la tasa de respuesta fue de $91 \%$, por lo que la posibilidad de un sesgo de selección es limitado; asimismo, se realizó un pequeño cuestionario con información básica para todos los niños invitados a participar y no se hallaron diferencias entre participantes y no participantes. Por último, otra limitación puede ser que, dado que se trató de un estudio transversal, no es posible diferenciar si el consumo de ciertos alimentos cambió por la ocurrencia de la enfermedad (asma), es decir, si la exposición antecede al episodio.

Existe la posibilidad de que se presente un sesgo de memoria, dado que se emplea un cuestionario y las madres de los niños con asma o rinitis efectuaron quizá un mayor esfuerzo en recordar el tipo de alimentación de sus hijos que las madres cuyos niños no presentaron estos problemas. Sin embargo, esto es poco probable porque los participantes no conocían la hipótesis del estudio.

Otra posibilidad en estudios transversales es que las madres de niños asmáticos cambien la alimentación de sus hijos por el hecho de tener síntomas; pese a ello, además de la nuez que actúa como alergeno, si los niños con síntomas tienen un consumo incrementado de frutas y verduras mantienen una relación positiva y un efecto protector de estos alimentos.

Es importante señalar que se utilizó una metodología estandarizada (ISAAC) para investigar las principales variables: asma, asma actual, gravedad del asma y síntomas nasales, por lo que se redujo la probabilidad de una mala clasificación.

En resumen, es posible concluir que los resultados proveen evidencia de que la dieta juega un papel muy importante en el asma y la rinitis. El consumo elevado de comida rápida y golosinas, como papas fritas y chocolate, tiene un efecto negativo sobre la salud de los niños que padecen dichas enfermedades. Por otro lado, se encontró que el alto consumo de oleaginosas, pescado, mariscos, frutas y verduras tiene un efecto protector para las manifestaciones de asma y síntomas de rinitis. No obstante, los resultados tienen que confirmarse en estudios de tipo prospectivo, sobre dieta y asma, para establecer con claridad la función que desempeñan los alimentos en el desarrollo de las enfermedades estudiadas.

\section{Referencias}

I. Downs SH, Marks GB, Sporik R, Belosouva EG, Car NG, Peat JK. Continued increase in the prevalence of asthma and atopy.Arch Dis Child 200I;84:20-23.

2. Braman SS. The global burden of asthma. Chest 2006; 130:4S-12S. 3. Secretaría de Salud. Información epidemiológica. Morbilidad General México 2003. México, DF. [Consultado el I 8 de octubre de 2004].

Disponible en: http://www.dgepi.salud.gob.mx/infoepi

4. Taylor WR, Newacheck PW. Impact of childhood asthma on health. Pediatrics 1992;90:657-662.

5. Bener A, Janahi IA, Sabbah A. Genetics and environmental risk factors associated with asthma in schoolchildren. Allerg Immunol (Paris) 2005;37:163-168.

6. Bayram I, Güne er-Kendirli S,Yilmaz M,Altinta DU,Alparslan N, BingölKarakoç $\mathrm{G}$. The prevalence of asthma and allergic diseases in children of school age in Adana in southern Turkey. Turk J Pediatr 2004;46:22I-225.

7. Mallol J. Satellite symposium: asthma in the world. Asthma among children in Latin America. Allergol Immunopathol 2004;32: 100-103. 8. Schei MA, Hessen JO, Smith KR, Bruce N, McCracken J, Lopez V. Childhood asthma and indoor woodsmoke from cooking in Guatemala. J Expo Anal Environ Epidemiol 2004; 14: I I0-I I7.

9. Kurukulaaratchy R, Fenn M, Matthews S, Hasan Arshad S. The prevalence, characteristics of and early life risk factors for eczema in 10year-old children. Pediatr Allergy Immunol 2003; I4:I78-I83.

I0. Wong GW, Li ST, Hui DS, Fok TF, Zhong NS, Chen YZ, et al. Individual allergens as risk factors for asthma and bronchial hyperresponsiveness in Chinese children. Eur Respir J 2002;19:288-293.

II. Sarinho E, Schor D, Veloso MA, Rizzo JA. There are more asthmatics in homes with high cockroach infestation. Braz J Med Biol Res 2004;37: 503-5I0.

12. Montefort S, Lenicker HM, Caruna S, Agius Muscat H. Asthma, rhinitis and eczema in Maltese 13-15 year-old schoolchildren -- prevalence, severity and associated factors [ISAAC]. International Study of Asthma and Allergies in Childhood. Clin Exp Allergy 1998;28: 1089-1099. 13. Barraza-Villarreal A, Sanin-Aguirre LH, Tellez-Rojo MM, LacasañaNavarro M, Romieu I. Risk factors for asthma in school children from Ciudad Juarez Chihuahua. J Asthma 2003;40:413-423.

14. Huang SL, Lin KC, Pan WH. Dietary factors associated with physiciandiagnosed asthma and allergic rhinitis in teenagers: analyses of the first Nutrition and Health Survey in Taiwan. Clin Exp Allergy 200 I;31: 259-264.

15. Dunder T, Kuikka L, Turtinen J, Räsänen L, Uhari M. Diet, serum fatty acids, and atopic diseases in childhood. Allergy 2001;56:425-428. 16. Bolte G, Frye C, Hoelscher B, Meyer I,Wjst M, Heinrich J. Margarine consumption and allergy in children. Am J Respir Crit Care Med 200I;163:277-279.

17. Antova T, Pattenden S, Nikiforov B, Leonardi GS, Boeva B, Fletcher T, et al. Nutrition and respiratory health in children in six Central and Eastern European countries. Thorax 2001;58:23I-236.

18. Romieu I,Varraso R, Avenel V, Leynaert B, Kauffmann F, ClavelChapelon F. Fruit and vegetable intakes and asthma in the E3N study. Thorax 2006;61:209-215.

19. Forastiere F, Pistelli R, Sestini P, Fortes C, Renzoni E, Rusconi F, et al. Consumption of fresh fruit rich in vitamin $C$ and wheezing symptoms in 
children. SIDRIA Collaborative Group, Italy (Italian Studies on Respiratory Disorders in Children and the Environment). Thorax 2000;55:283-288.

20. Cook DG, Carey IM, Whincup PH, Papacosta O, Chirico S, Bruckdorfer $\mathrm{KR}$, et al. Effect of fresh fruit consumption on lung function and wheeze in children. Thorax 1997;52:628-633.

2I. Ellwood P,Asher MI, Björkstén B, Burr M, Pearce N, Robertson CF. Diet and asthma, allergic rhinoconjunctivitis and atopic eczema symptom prevalence: an ecological analysis of the International Study of Asthma and Allergies in Childhood (ISAAC) data. ISAAC Phase One Study Group. Eur Respir J 200I; 17:436-443.

22. Hijazi N,Abalkhail B, Seaton A. Diet and childhood asthma in a society in transition: a study in urban and rural Saudi Arabia. Thorax 2000;55: 775-779.

23.Wijga AH, Smit HA, Kerkhof M, de Jongste JC, Gerritsen J, Neijens HJ, et al.Association of consumption of products containing milk fat with reduced asthma risk in pre-school children: the PIAMA birth cohort study. Thorax 2003;58:567-572.

24. Hodge L, Salome CM, Peat JK, Haby MM, Xuan W, Woolcock A. Consumption of oily fish and childhood asthma risk. Med J Aust 1996; 164:137-140.

25. Nafstad P, Nystad W, Magnus P, jaakkola J].Asthma and allergic rhinitis at 4 years of age in relation to fish consumption in infancy. J Asthma 2003;40:343-348

26. Riedler J, Braun-Fahrländer C, Eder W, Schreuer M, Waser M, Maisch $S$, et al. Exposure to farming in early life and development of asthma and allergy: a cross-sectional survey. Lancet 200 I;358: I 29- I 33 .

27. Black PN, Sharpe S. Dietary fat and asthma: is there a connection? Eur Respir J 1997; 10:6-12.

28. Hodge L, Salome CM, Hughes JM, Liu-Brennan D, Rimmer J, Allman $M$, et al. Effect of dietary intake of omega- 3 and omega- 6 fatty acids on severity of asthma in children. Eur Respir J 1998; | I:361-365.

29. Coordination Committee for the International Study of Asthma and Allergies in Childhood (ISAAC). Manual for the International Study for Asthma and Allergies in Childhood (ISAAC). Bochum (FRG), 1992. [Consultado el 25 de octubre de 2005].Disponible en: http://isaac. auckland.ac.nz/phases/phaseone/phaseonemanual.pdf 30 Asher MI, Keil U,Anderson HR, Beasley R, Crane J, Martinez F, et al. International Study of Asthma and Allergies in Childhood (ISAAC): rationale and methods. Eur Respir J 1995;8:483-491.
31. Tatto-Cano MI, Sanín-Aguirre LH, González V, Ruiz-Velasco S, Romieu I. Prevalencia del asma, rinitis, y ezcema en niños escolares de Cuernavaca, Morelos. Salud Publica Mex 1997;39:497-506.

32. Canseco C, Leal L, Mora H, Galindo G. Epidemiología de las enfermedades alérgicas en el área metropolitana de Monterrey. Rev Alerg Mex 1991;38:95-101.

33. Barraza-Villarreal A, Sanín-Aguirre LH,Téllez-Rojo MM, LacasañaNavarro M, Romieu I. Prevalencia de asma y otras enfermedades alérgicas en niños escolares de Ciudad Juárez, Chihuahua. Salud Publica Mex 200I;43:433-443.

34. Wong GW, Ko FW, Hui DS, Fok TF, Carr D, von Mutius E, et al. Factors associated with difference in prevalence of asthma in children from three cities in China: multicentre epidemiological survey. BMJ 2004;329:486. 35. Garcia-Marcos L, Canflanca IM, Garrido JB,Varela AL, GarciaHernandez G, Guillen Grima F, et al. Relationship of asthma and rhinoconjunctivitis with obesity, exercise and Mediterranean diet in Spanish schoolchildren. Thorax 2007;62:503-508.

36. Shin NH, Ryu SY, Lee H, Min KR, Kim Y. Inhibitory effects of hidroxystilbenes on cicloxigenase from sheep seminal vesicles. Planta Med 1998;64:283-284.

37. Crosby GA. Lignans in food and nutrition. Food Technol 2005;59:32-35. 38. Romieu I,Trenga C. Diet and obstructive lung diseases. Epidemiol Rev 200I;23:268-287.

39. Troisi RJ,Willett WC,Weiss ST, Trichopoulos D, Rosner B, Speizer FE.A prospective study of diet and adult-onset asthma. Am J Respir Crit Care Med 1995; I5I:I40I-I408.

40. Gilliland FD, Berhane KT, LiYF, Kim DH, Margolis HG. Dietary magnesium, potassium, sodium, and children's lung function. Am J Epidemiol 2002; |55:125-|3|.

4I.Andreasyan K, Ponsonby AL, Dwyer T, Kemp A, Dear K, Cochrane J, et al.A differing pattern of association between dietary fish and allergenspecific subgroups of atopy.Allergy 2005;60:67I-677.

42. Muñoz de Chávez M. Los alimentos y sus nutrientes. Tablas de valor nutritivo de los alimentos. México DF: McGraw-Hill Internamericana, 2002. 43. Eriksson NE, Möller C, Werner S, Magnusson J, Bengtsson U, Zolubas M. Self-reported food hypersensitivity in Sweden, Denmark, Estonia, Lithuania, and Russia.J Investig Allergol Clin Immunol 2004;14:70-79. 